\title{
Status of the Baikal-GVD project
}

\begin{abstract}
A.V. Avrorin a , V.M. Aynutdinov ${ }^{\text {a }}$, R. Bannasch ${ }^{\text {h}}$, I.A. Belolaptikov ${ }^{\mathrm{c}}$, D.Yu. Bogorodsky ${ }^{\mathrm{b}}$, V.B. Brudanin ${ }^{\mathrm{c}}$, N.M. Budnev ${ }^{\text {b }}$, I.A. Danilchenko ${ }^{a}$, G.V. Domogatsky ${ }^{a}$, A.A. Doroshenko ${ }^{a}$, A.N. Dyachok ${ }^{b}$,

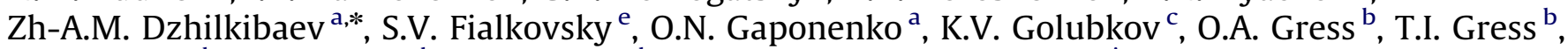
O.G. Grishin ${ }^{\text {b }}$, K.G. Kebkal ${ }^{\text {h}}$, A.G. Kebkal ${ }^{\text {h }}$, A.M. Klabukov ${ }^{\text {a }}$, A.I. Klimov ${ }^{\text {i }}$ K.V. Konishchev ${ }^{\text {c }}$,
\end{abstract} A.V. Korobchenko ${ }^{\text {}}$, A.P. Koshechkin ${ }^{\text {a }}$, F.K. Koshel ${ }^{a}$, V.A. Kozhin ${ }^{d}$, V.F. Kulepov ${ }^{\text {e, D.A. Kuleshov }}{ }^{\text {a }}$, L.A. Kuzmichev ${ }^{\text {d, }}$, V.I. Ljashuk $^{\text {a }}$, S.P. Mikheev ${ }^{\text {a }}$, M.B. Milenin ${ }^{\text {e }}$, R.A. Mirgazov ${ }^{\text {b }}$, E.R. Osipova ${ }^{d}$, A.L. Pakhorukov $^{\text {b }}$, A.I. Panfilov ${ }^{\text {a }}$, A.L. Pan'kov ${ }^{\text {b }}$, L.V. Pan'kov ${ }^{\text {b }}$, A.A. Perevalov ${ }^{\text {b. D.A. Petukhov }}{ }^{\text {a }}$, E.N. Pliskovsky ${ }^{\text {c }}$, V.A. Poleshuk ${ }^{\text {b }}$, E.G. Popova ${ }^{\text {d }}$, M.I. Rozanov ${ }^{g}$, V.F. Rubtsov ${ }^{\text {b }}$, E.V. Rjabov ${ }^{\text {b }}$, B.A. Shaybonov ${ }^{\text {c }}$, A.V. Shirokov ${ }^{\text {d }}$, A.A. Sheifler ${ }^{c}$, A.V. Skurikhin ${ }^{d}$, Ch. Spiering ${ }^{f}$, O.V. Suvorova ${ }^{a}$, B.A. Tarashchansky ${ }^{\text {b }}$, A.S. Yagunov ${ }^{\text {b }}$, A.V. Zagorodnikov ${ }^{\text {b }}$, V.A. Zhukov ${ }^{\text {a }}$, V.L. Zurbanov ${ }^{\text {b }}$

\footnotetext{
a Institute for Nuclear Research, Moscow 117312, Russia

${ }^{\mathrm{b}}$ Irkutsk State University, Irkutsk 664003, Russia

c Joint Institute for Nuclear Research, Dubna 141980, Russia

d Institute of Nuclear Physics, Moscow State University, Moscow 119991, Russia

e Nizhni Novgorod State Technical University, Nizhni Novgorod 603950, Russia

${ }^{\mathrm{f}}$ DESY, Zeuthen D-15738, Germany

${ }^{g}$ St. Petersburg State Marine Technical University, St. Petersburg 190008, Russia

${ }^{\mathrm{h}}$ EvoLogics GmbH, Berlin, Germany

${ }^{\text {i }}$ Russian Research Center Kurchatov Institute, Moscow 123182, Russia
}

\section{A R T I C L E I N F O}

\section{Available online 24 January 2012}

\section{Keywords:}

Neutrino

Neutrino telescopes

Baikal

\begin{abstract}
A B S T R A C T
The construction of a $\mathrm{km}^{3}$-scale neutrino telescope - the Gigaton Volume Detector (GVD) in Lake Baikal - is the central goal of the Baikal collaboration. During the R\&D phase of the GVD project in 2008-2010 years the basic elements of GVD - new optical modules, FADC readout units, underwater communications and trigger systems - have been developed, produced and tested in situ by long-term operating prototype strings in Lake Baikal. The prototyping phase of the GVD project has been started in April 2011 with the installation of a three string array (prototype cluster) which comprises all basic elements and systems of the GVD-telescope in Lake Baikal. We describe configuration and technical design of the GVD, present selected results obtained during 2008-2010 with prototype strings, and describe configuration and design of the 2011 prototype cluster.
\end{abstract}

(c) 2012 Elsevier B.V. All rights reserved.

\section{Introduction}

The Baikal collaboration follows since several years a R\&D program for a Gigaton Volume Detector (GVD) in Lake Baikal. GVD will be a kilometer-scale high-energy neutrino observatory $[1,2]$. The main scientific goal of GVD is to map the high-energy neutrino sky in the Southern Hemisphere including the region of the galactic center. Other topics include the indirect search for dark matter by searching for neutrinos produced in WIMP annihilation in the Sun or in the center of the Earth. GVD will

\footnotetext{
* Corresponding author.

E-mail address: djilkib@yandex.ru (Zh.-A.M. Dzhilkibaev).
}

also search for exotic particles like magnetic monopoles, supersymmetric Q-balls or nuclearites.

The GVD detector will be located in the southern basin of Lake Baikal close to the NT200+ telescope $[3,4]$. The geographical coordinates of the detector site are $51^{\circ} 50^{\prime} \mathrm{N}$ and $104^{\circ} 20^{\prime} \mathrm{E}$. Since the slope of the shore bottom relief is rather steep, the telescope can be arranged comparatively nearly to shore at distances of 4$5 \mathrm{~km}$. The depth of the lake is about $1400 \mathrm{~m}$ at this place. The light propagation in the Baikal water is characterized by an absorption length of $20-25 \mathrm{~m}$ and a scattering length of $30-50 \mathrm{~m}$. The water luminescence is low at the detector site. The rate of light pulses from $\mathrm{K}^{40}$-decays is negligible.

The first generation Baikal Neutrino Telescope NT200 is operating in Lake Baikal since April 1998 [5-8]. NT200 consists 
of eight $72 \mathrm{~m}$ long strings, each with 24 pairwise arranged optical modules (OMs). Each OM contains a $37-\mathrm{cm}$ diameter hybrid photodetector QUASAR-370, developed specially for this project [9]. The upgraded Baikal telescope NT200+ [3] was commissioned in April 2005, and consists of a central part (the former, densely instrumented NT200 telescope) and three additional external strings. The construction of NT200+ was a first step towards a $\mathrm{km}^{3}$-scale neutrino telescope. The important $\mathrm{km}^{3}$ milestones were the construction and installation of prototype strings in 2009-2010 [1,10] and a prototype GVD cluster, comprising three strings, in 2011. The basic goals of the prototype array installation are investigation and in situ test of basic elements of the future detector. In this paper we review the R\&D activities towards a $\mathrm{km}^{3}$-scale Baikal telescope.

\section{GVD design}

In this section we will discuss basic principles of the GVD design and the results of optimization of the GVD configuration.

The basic approach designing the Baikal-GVD is the same as for the NT200 design. This approach provides a relatively flexible structure, which allows for a future expansion of the instrumented volume as well as a rearrangement of the main building blocks (clusters), to adapt to requirements of new scientific goals, if necessary.

GVD will consist of strings of optical modules (OMs) (see, Fig. 1). Each string will include a chain of OMs spaced uniformly at depths $900-1250 \mathrm{~m}$. A conventional string structure is optimal

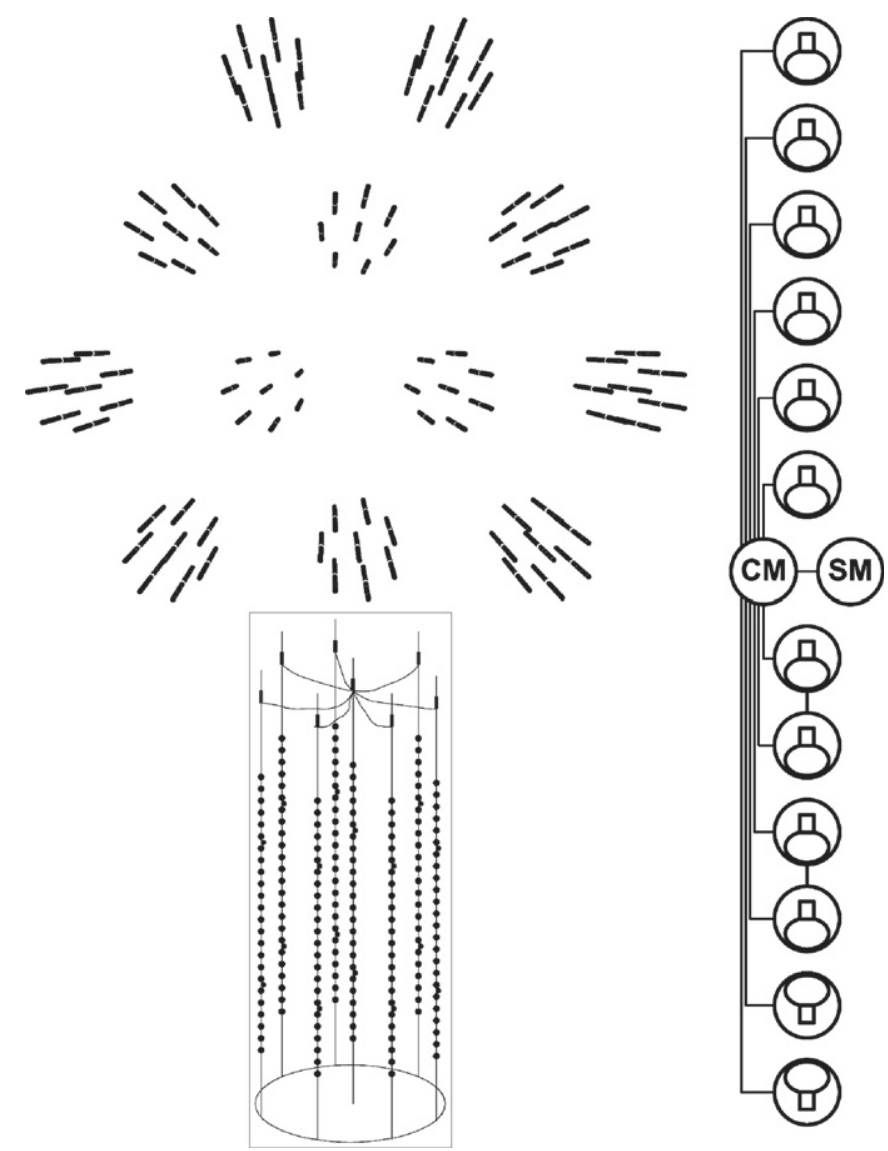

Fig. 1. GVD design: GVD top view (12 clusters); schematic view of cluster (eight strings with two sections each); and a section of OMs (12 OMs with PMTs R7081HQE). for telescope deployment from the natural ice platform of Lake Baikal. For interstring communication and connection to shore, strings will be grouped in clusters. Each cluster will be controlled by the cluster DAQ center placed near the water surface. The DAQ of the cluster has a flexible structure, which allows forming clusters with different number of strings and optical modules. The present version of the DAQ relates to an eight-string cluster with 24 optical modules on each string.

\subsection{Optical module}

The optical module is the basic element of the future GVD neutrino telescope. Each OM (see, Fig. 2) contains a photomultiplier tube (PMT), which detects the Cherenkov light produced by relativistic charged particles passing through the water. The information from the ensemble of OMs allows reconstruction of event topology and energy. After testing different options the photomultiplier Hamamatsu R7081HQE was selected as a light sensor. This PMT has a hemispherical photocathode with 10 in. diameter and quantum efficiency up to $35 \%$. The functional scheme of the optical module electronics is presented in detail in [2].

Besides the PMT, an OM comprises a high voltage power supply unit (HV), a fast two-channel preamplifier, and a controller. The HV unit (Traco Power SHV 12-2.0 K $1000 \mathrm{P}$ ) provides the power for the phototube divider (18 $\mathrm{M} \Omega$ resistance) in the range from 0 up to $2 \mathrm{kV}$. The tube gains have been adjusted to about $10^{7}$. This gain is provided by divider voltages between 1250 and $1650 \mathrm{~V}$, depending on the individual tube. An additional signal amplification by a factor of 10 is provided by the first channel of the preamplifier. This gain value results in single photoelectron pulse amplitudes of $30-40 \mathrm{mV}$ in average. This corresponds to a spectrometric channel linearity range up to about 100 photoelectrons. The second preamplifier output with factor 20 is intended for PMT noise monitoring. For time and amplitude calibration of the measuring channel, two LEDs are installed in the optical module. The dominant wavelength of the LED is $445 \mathrm{~nm}$, the LED pulse has a width of about $5 \mathrm{~ns}$ (FWHM). The possibility of independent regulation of the LED light intensity and low cross talk between LED channels $(<1 \%)$ allow to directly measure the linearity range of the spectrometric channel.

The OM controller is intended for HV regulation and monitoring, for PMT noise measurements, and for time and amplitude calibration. The OM controller is designed on the basis of the

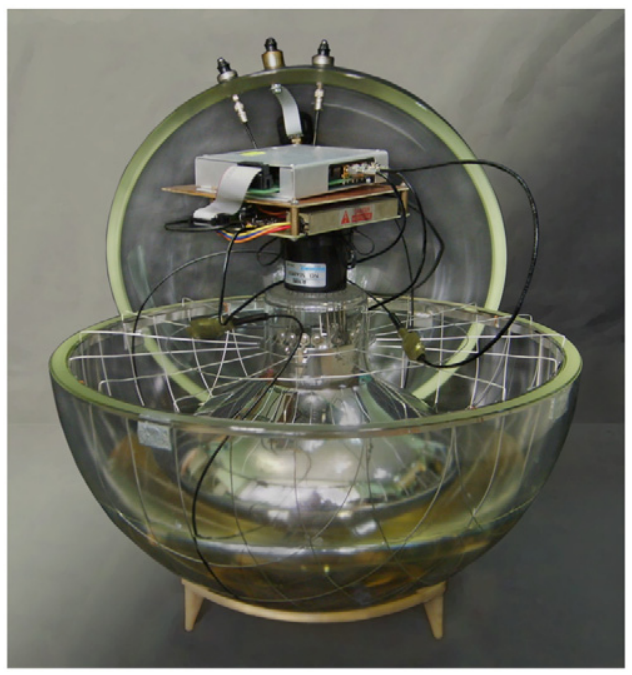

Fig. 2. GVD optical module. 


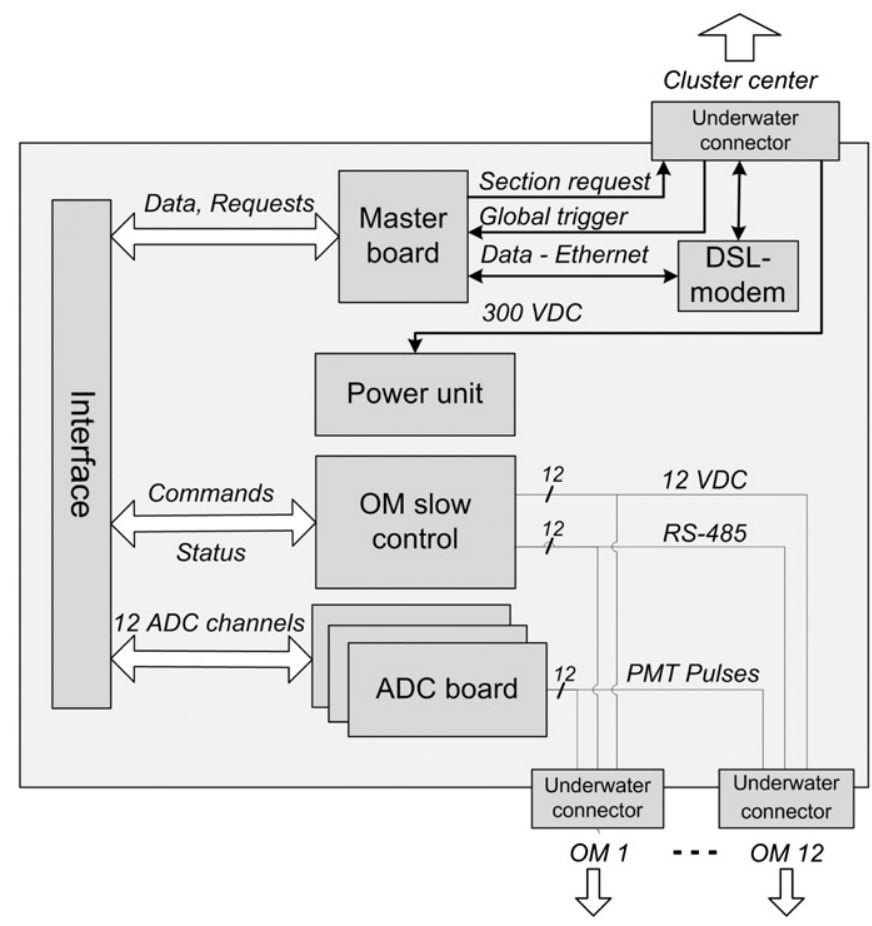

Fig. 3. Block diagram of the string section center module (CM).

micro-controller SiLabs C8051F121. Slow control data to and from the OMs are transferred via an underwater RS-485 bus.

The OM electronics and the PMT are placed in a pressureresistant glass sphere VITROVEX with $42 \mathrm{~cm}$ diameter. A high permittivity alloy cage surrounds the PMT, shielding it against Earth's magnetic field.

\subsection{The string section}

The optical modules on a string are grouped into two the lowest-level DAQ units. Each section includes 12 OMs and the central module CM (see, Fig. 3). PMT signals from all OMs of a section are transmitted to the $\mathrm{CM}$ trough $90 \mathrm{~m}$ long coaxial cables, where they are digitized by custom-made four-channel ADC boards with $200 \mathrm{MHz}$ sampling rate. The time bin value of 5 ns provides opportunity for PMT pulse area and leading edge position measurements (passing $90 \mathrm{~m}$ long coaxial cable, the width of signal increases up to 20 ns (FWHM)). The corresponding time accuracy of the measuring channel is less than 2 ns.

The CM consists of three ADC boards, an OM slow-control unit, and a Master board.

The OM slow-control unit provides data communication between OM controllers and Master board via an underwater RS-485 bus. Also, this unit is intended for the OM power control (to switch power on/off for each optical module independently).

Each channel of an ADC board (see, Fig. 4) includes a fast digitizer on the basis of AD9430 microcircuits with 12 bit resolution. The digitized signals from each ADC are transferred to a FPGA (Xilinx Spartan 3) which handles the data. A memory buffer of $12 \mathrm{kB}$ allows to accumulate waveform data from the ADC for $30 \mu \mathrm{s}$. Two options of memory organization are possible: single or double-buffered memory. A double-buffered memory is minimizing the readout dead-time: while one buffer is ready for readout, the second one is connected to the ADC output.

The trigger signal from the Master board stops the buffer accumulation, and the waveform information is transferred to the Master board through the interface board. The beginning and the length of the waveform interval can be configured. Each ADC data

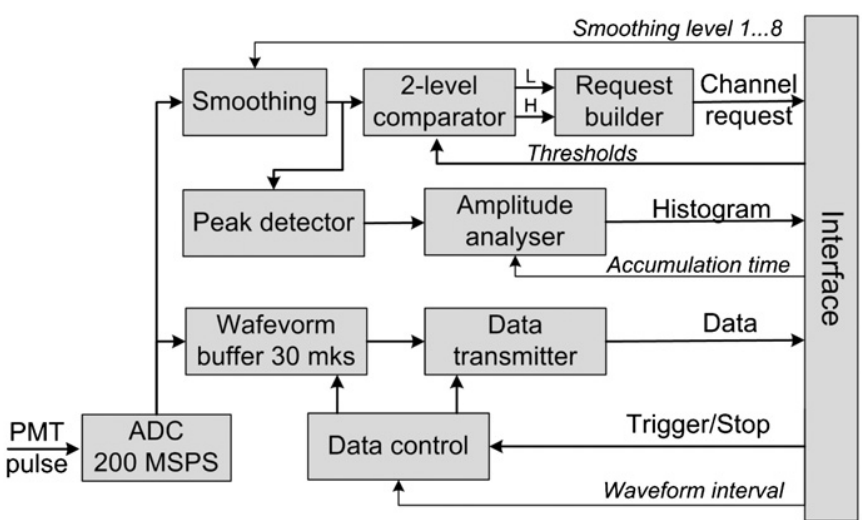

Fig. 4. Block diagram of the ADC board (one channel) of the string section center module.

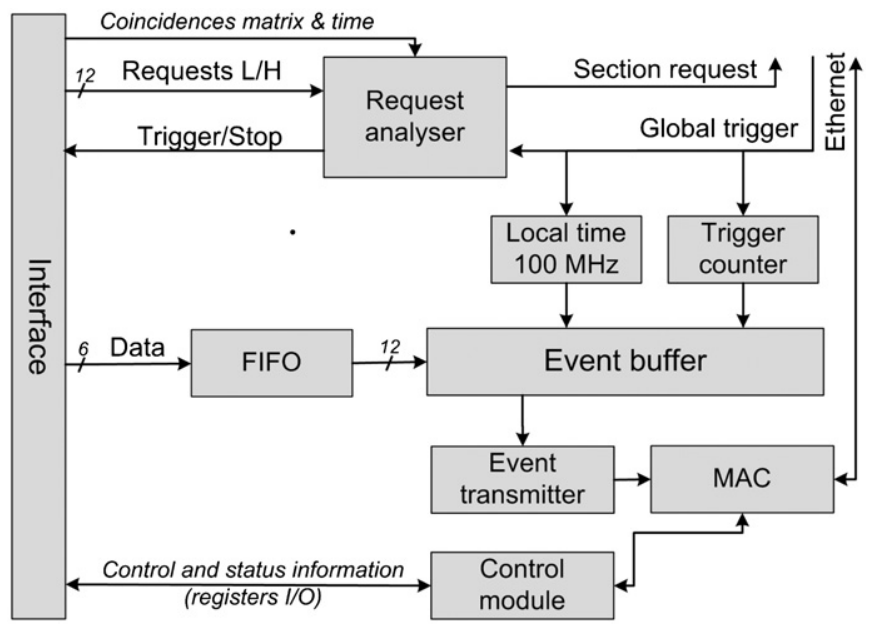

Fig. 5. Block diagram of the Master board of the string section center module.

channel is also connected to a peak detector and amplitude analyzer, which accumulates monitor histograms in a programmable time interval. This information is intended for the monitoring of the channel trigger rate. An ADC trigger request channel includes a smoothing unit (smoothing level from 1 to 8 ) for electronics noise reduction, a two-level adjustable digital comparator (low threshold $L$ and high threshold $H$ ), and a request builder, which build the request to the trigger logic. The requests $L$ or $H$ are transferred to the Master board.

The block diagram of the Master board is shown in Fig. 5. The Master board provides trigger logic, data readout from ADC boards, connection via local Ethernet to the cluster DAQ center, and control of the section operation. The request analyzer forms the section trigger request (local trigger) on the basis of requests $L$ and $H$ from 12 ADC channels. This unit contains a programmable coincidence matrix $(12 \mathrm{H} \times 12 \mathrm{~L})$, which provides a simple way to generate the section trigger request. The basic trigger modes are: (A) coincidences of $>N L$-requests within a selectable time window ( $L>N$-trigger), or (B) coincidences of $L$ and $H$ requests from any neighbouring OMs within a section (L\&H-trigger).

The section trigger request is transferred to the cluster DAQ center, where a global trigger for all sections is formed. The global trigger produces the stop signal for all ADC channels and initiates waveform information readout. The time delay of the global trigger is about $15 \mu \mathrm{s}(2 \mathrm{~km} \times 1.2 \mathrm{~km}$ of the string cable and electronics delay). Waveform information is accumulated in the event buffer $(512 \mathrm{kB})$. Each event contains waveform data for all ADCs of the section, the global trigger number, and the local time 
based on a $100 \mathrm{MHz}$ clock. The event trigger number provides the possibility of event synchronization for the different sections. Data from the event buffer are transmitted via an Ethernet connection to the cluster DAQ center.

The control module provides access to the $\mathrm{I} / \mathrm{O}$ registers of the Master and ADC boards and the control for the data transmission via RS-485 bus.

\subsection{Optimization of GVD configuration}

The objective of the optimization of the GVD configuration is to provide a large cascade detection volume with the condition of also effectively recording high energy muons. The MC-optimization for the $\mathrm{km}^{3}$-detector configuration was performed for 96 strings grouped in 12 clusters with 192 OMs each. Basic parameters for the optimization were: $Z$-the vertical distance between OMs, $R$ - the distance between strings and cluster center, and $H$-the distance between neighbouring clusters centers. Coincidences of any neighbouring OMs on a string were used as an initial trigger condition.

In a case of showers at least 15 hit OMs on at least three strings (15/3-trigger) were required for event selection. It was shown that the effective volume weakly depends on the values of $Z$ and $R$ parameters. Fig. 6 illustrates the dependence of effective volume on the distance $H$ between neighbouring clusters. Curves in Fig. 6 from top to bottom correspond to $H \rightarrow \infty$ (12 independent clusters), $H=300 \mathrm{~m}$, and $H=200 \mathrm{~m}$, respectively. The effective volume increases with increasing distance $H$ between clusters.

In case of muons at least six hit OMs on at least three strings (6/3 condition) were required for event selection. Results of MCcalculations show that muons effective area increases with $R$ decreasing in the range of $(40 / 100) \mathrm{m}$, and weakly depends on the values of $Z$ and $H$ parameters for given fixed value of $R$. The energy dependence of muon effective areas for different configurations are shown in Fig. 7. Preferable configurations correspond to parameters $H=250-300 \mathrm{~m}, R=40-60 \mathrm{~m}$, and $Z=15 \mathrm{~m}$.

An optimum for cascade detection volume and muon effective area was obtained for the following values of the parameters: $H=300 \mathrm{~m}, R=60 \mathrm{~m}$, and $Z=15 \mathrm{~m}$. The design of GVD is presented in Fig. 1. The neutrino detector will consist of $2304 \mathrm{OMs}$, arranged on 96 strings with instrumented length of $345 \mathrm{~m}$ (24 OMs on each string).

The GVD effective area for muons (6/3 condition) with an arrival direction reconstruction error about of $0.5^{\circ}$ (median value)

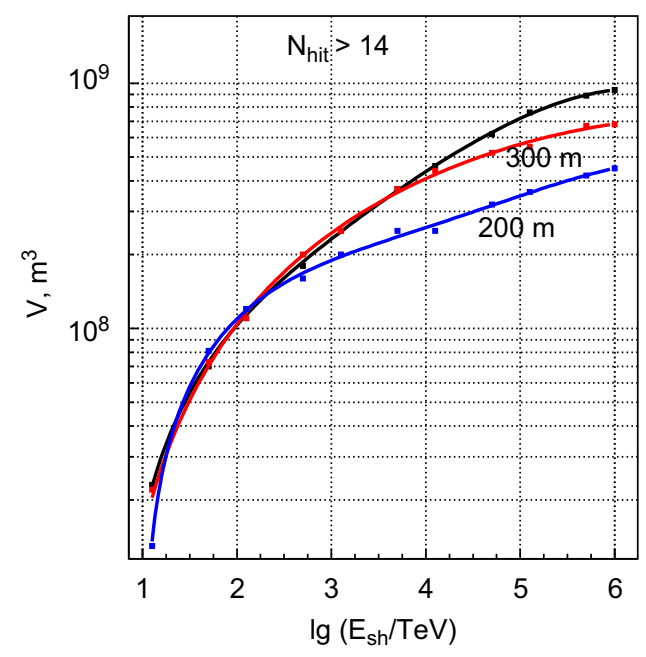

Fig. 6. GVD shower detection volume (15/3 condition).

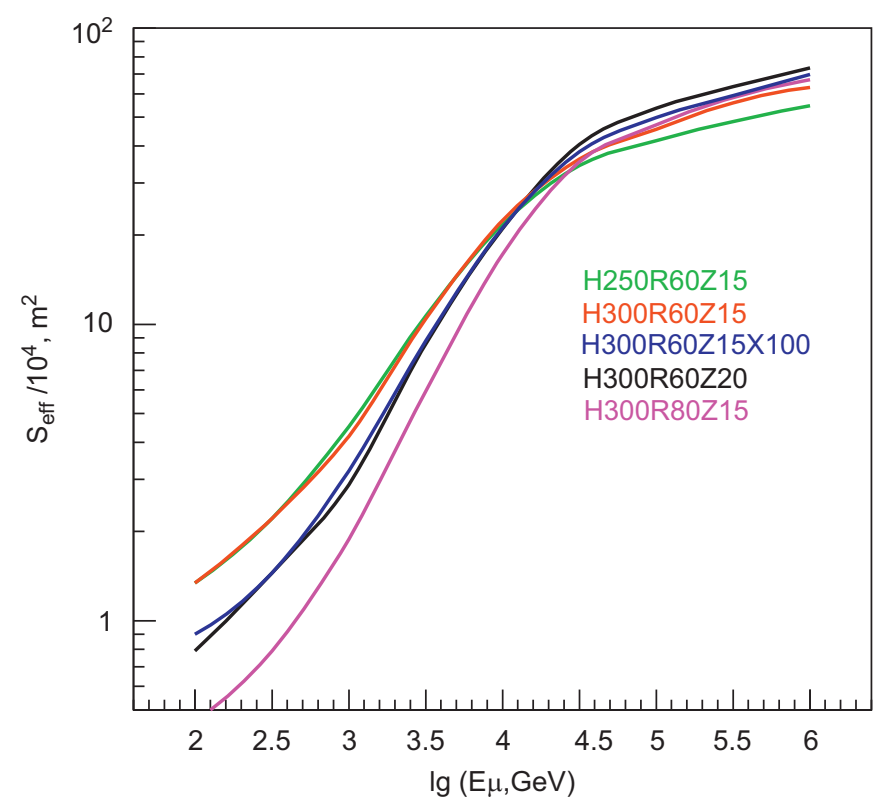

Fig. 7. GVD muon effective area (6/3 condition).

and energy resolution of $\delta \lg E \sim 0.4$ rises from $0.1 \mathrm{~km}^{2}$ at $3 \mathrm{TeV}$ to $0.8 \mathrm{~km}^{2}$ asymptotically.

The effective volume for cascades (15/3 condition) above $100 \mathrm{TeV}$ with reconstruction error about $5-7^{\circ}$ (median values) and energy resolution of about (10-15)\% rises from 0.1 to $0.7 \mathrm{~km}^{3}$.

We note that the effective areas and volumes given above apply to the defined condition levels. We are presently working on the optimization of cuts to reject background from downward muons, including those from accidentally coinciding air showers. The corresponding cuts will reduce the effective area and volumes.

\section{Prototype arrays}

\subsection{Prototypes of GVD string}

The first prototype of GVD electronics was installed in Lake Baikal in April 2008. It was a reduced-size section with six OMs. This unit provided the possibility to study basic elements of the future detector: new optical modules and FADC based measuring system. During the next two years different versions of prototype string were tested in Lake Baikal as a part of NT200+ detector. The prototype string 2009 consists of 12 optical modules with six photomultipliers R8055 and six XP1807. In April 2010, a prototype of the GVD string with eight PMTs R7081HQE and four PMTs R8055 was installed in Lake Baikal.

In situ tests of the prototype strings allow to estimate the accuracy with which the system can determine the arrival time of a photon at the photocathode. The measurements were performed with the LED flasher, laser calibration source and cosmic ray muons. The first step of data analysis was a study of the accuracy of the pulse time measurement with delayed pulses produced by the LED flasher. The arrival time of a photon is determined from the waveform data. We find the average delay values obtained from waveform data to be close to the nominal value (average deviation $\sim 1 \mathrm{~ns}$ ). The average RMS is less than 2 ns. This value characterizes the time resolution of the measuring channels.

More detailed studies of the time accuracy were performed with a laser source located at a distance of about $100 \mathrm{~m}$ from the 
prototype string. The time differences between pairs of channels measured for laser pulses are in good agreement with the expected values. The average deviation is about 2 ns. This estimation takes into account time calibration errors and string position uncertainties.

The atmospheric muon flux is a natural calibration source which allows to test the performance of the array measuring systems, as well as to estimate the efficiency of background suppression and event reconstruction procedures. Prototype string data allow to reconstruct the zenith angle distribution of downward going atmospheric muons. A selected sample of 2010 prototype string data was used for the atmospheric muons analysis. The typical level of OMs counting rates corresponding to this data sample is shown in Fig. 8. A sample of MC-events from atmospheric muons has been generated (see, for details $[5,6,11]$ ), taking into account the features of prototype string measuring system and actual counting rates of optical modules.

At the first step of analysis a causality criterion, as well as a special muon selection conditions were applied to events for elimination of background signals caused by PMTs and water luminescence noise, muon bundles and electro-magnetic showers induced by muons. At the next step the cleaned time information of OMs was used for track reconstruction with a trigger condition $>3$ hit OMs. Finally, soft cuts on $\chi^{2}$ value and on the error of reconstructed zenith angle were applied for muon event selection. Fig. 9 illustrates the precision of the zenith angle reconstruction procedure. Shown here is a distribution of mismatch angles between generated and reconstructed zenith angles of muons. An

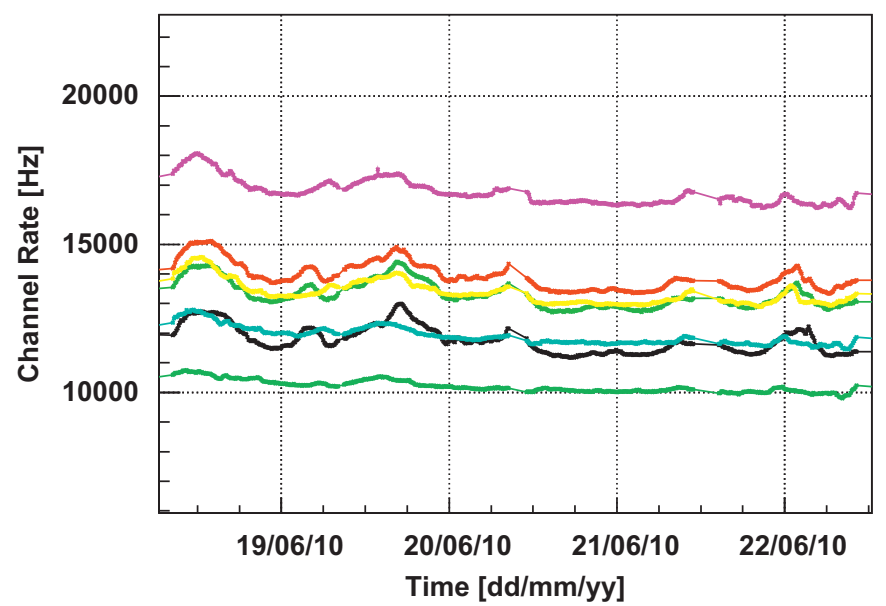

Fig. 8. Counting rates of seven different optical modules with R7081HQE PMTs of 2010 prototype string.

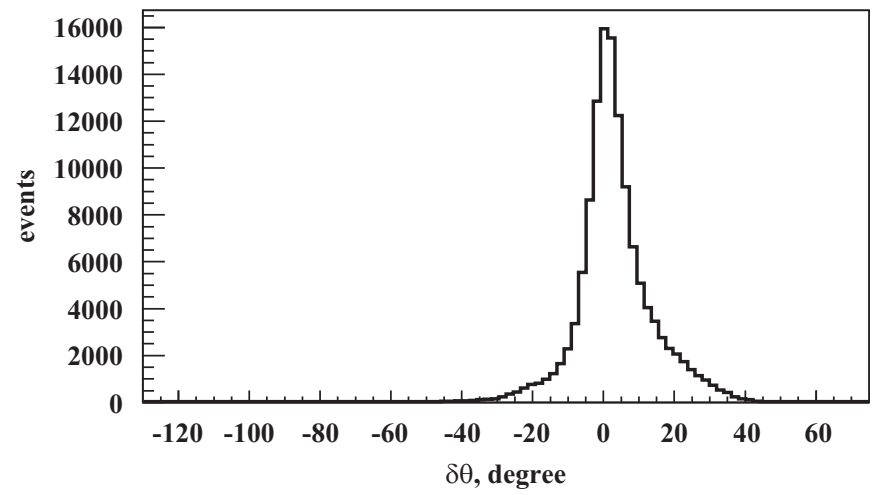

Fig. 9. Distribution of mismatch angles after final cuts. $\delta \theta$-difference between generated and reconstructed zenith angles. expected average number of muons in finally selected events is about 1.8. This explains the rather wide shape of mismatch angle distribution in Fig. 9. Zenith angular distributions of experimental and MC-simulated event rates are shown in Fig. 10. The good consistency between the data and theoretical expectation confirms the expected performance of the time measuring systems and the efficiency of used calibration methods, as well as the efficiency of event selection and noise suppression procedures.

\subsection{Prototype of GVD cluster}

The operation of GVD prototype strings in 2009 and 2010 allows a first assessment of the DAQ performance. On the basis of the experience of prototype string operation, in April 2011 a prototype GVD cluster with three strings was installed in Lake Baikal and put in operation. Each string consists of one section with eight optical modules. Distances between the OMs are $10 \mathrm{~m}$ along the string; distances between the strings are $40 \mathrm{~m}$. The OMs house photomultipliers of different types: 16 PMT R7081HQE, three PMT XP1807, and five PMT R8055. Also, transmitters and receivers of two alternative acoustic positioning systems are arranged on each string. A sketch of prototype cluster and layout of cluster, NT200+ and communication lines is shown in Figs. 11 and 12 .

Analog pulses from PMTs of the string section are transferred to the central modules (CMs). The configuration of the central module was presented in Fig. 3. For the connection between the $\mathrm{CM}$ and the cluster center an armored $1.2 \mathrm{~km}$ carrier cable was deployed (custom designed for this setup). This cable includes two coaxial lines for request and global trigger signals, a screened twisted pair for the DSL-modem data channel, and three power lines.

The cluster DAQ center is placed near the surface $(\sim 30 \mathrm{~m}$ depth as for GVD layout). It provides the string triggering, power supply, and communication to shore. A block diagram of the cluster center electronics is presented in Fig. 13. The organizations of central and section trigger systems are the same. The section request lines are connected to three inputs of the central $\mathrm{ADC}$ board. The request pulse waveforms are accumulated in the ADC buffers. The Master board works out the global trigger for all strings. Data from the strings are transferred through DSL-modem Ethernet channel to the cluster center. The DSL-modem bandwidth was set to the level $4 \mathrm{Mb} / \mathrm{s}$ (about half of maximum data rate supported for $1.2 \mathrm{~km}$ cable line). This value restricts the maximum event rate per string section to about $25 \mathrm{~Hz}$. Each event has a fixed length of $16 \mathrm{kB}$ and contains waveform data for all ADC channels for a $5 \mu$ s time window centered around the trigger time.

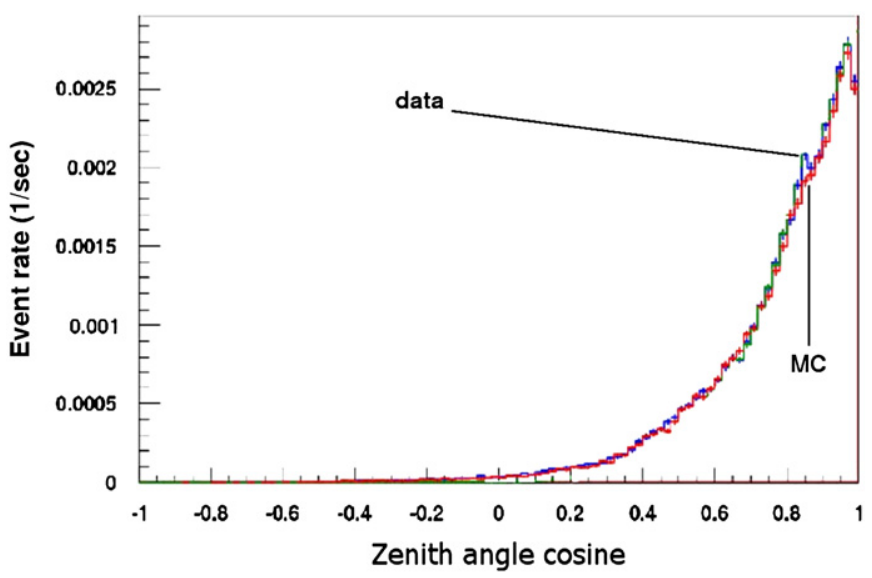

Fig. 10. Experimental and theoretically expected event rates after final cuts. 


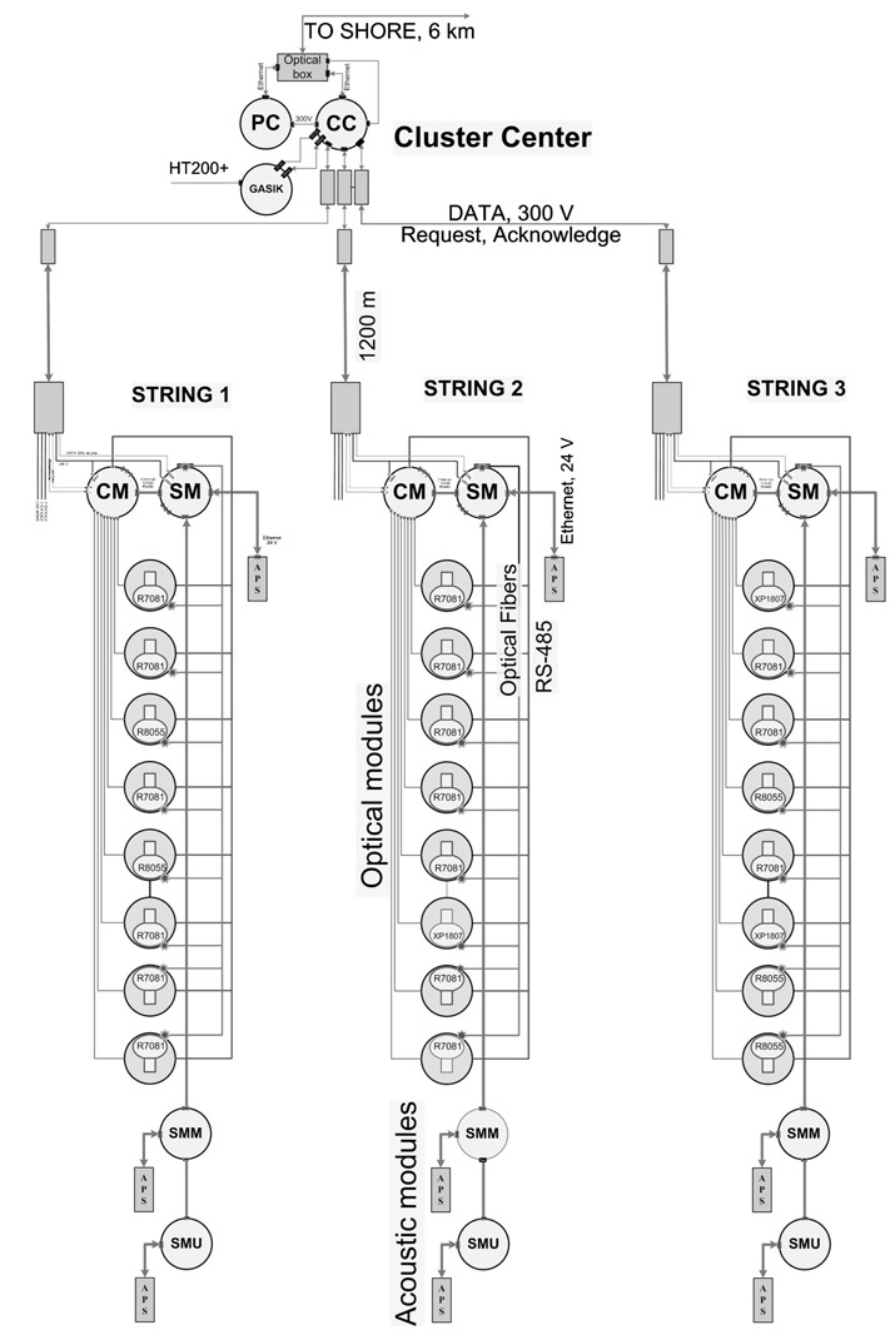

TO SHORE, $6 \mathrm{~km}$

(a)

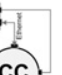

Fig. 11. Artistic view of the prototype cluster.

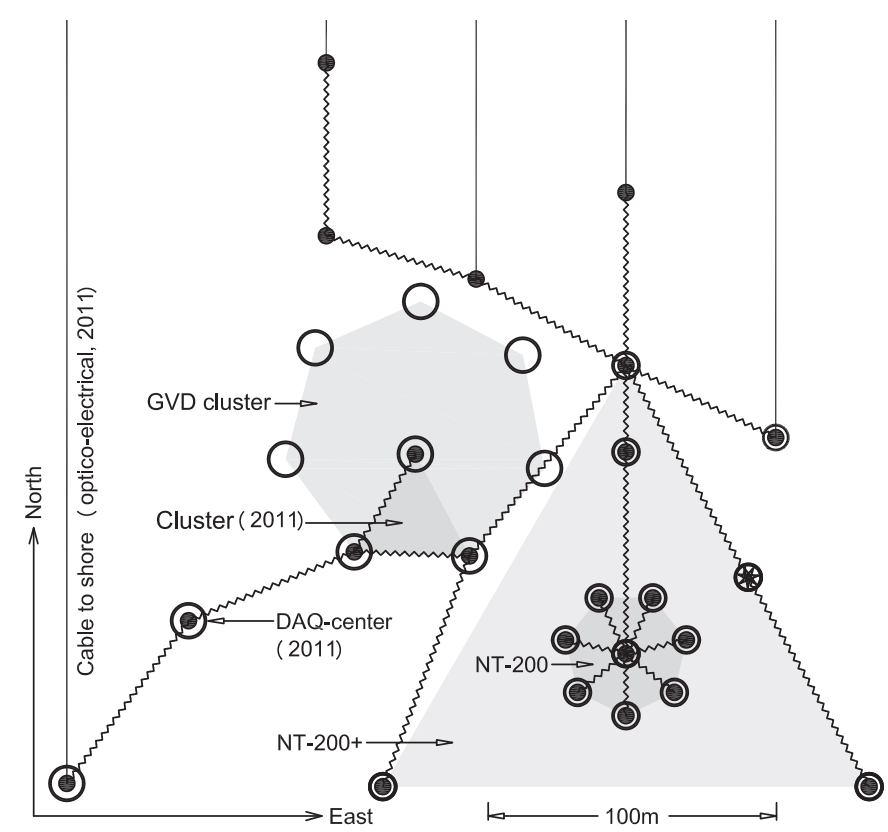

Fig. 12. Sketch of prototype cluster, neutrino telescope NT200+, and communication lines locations.

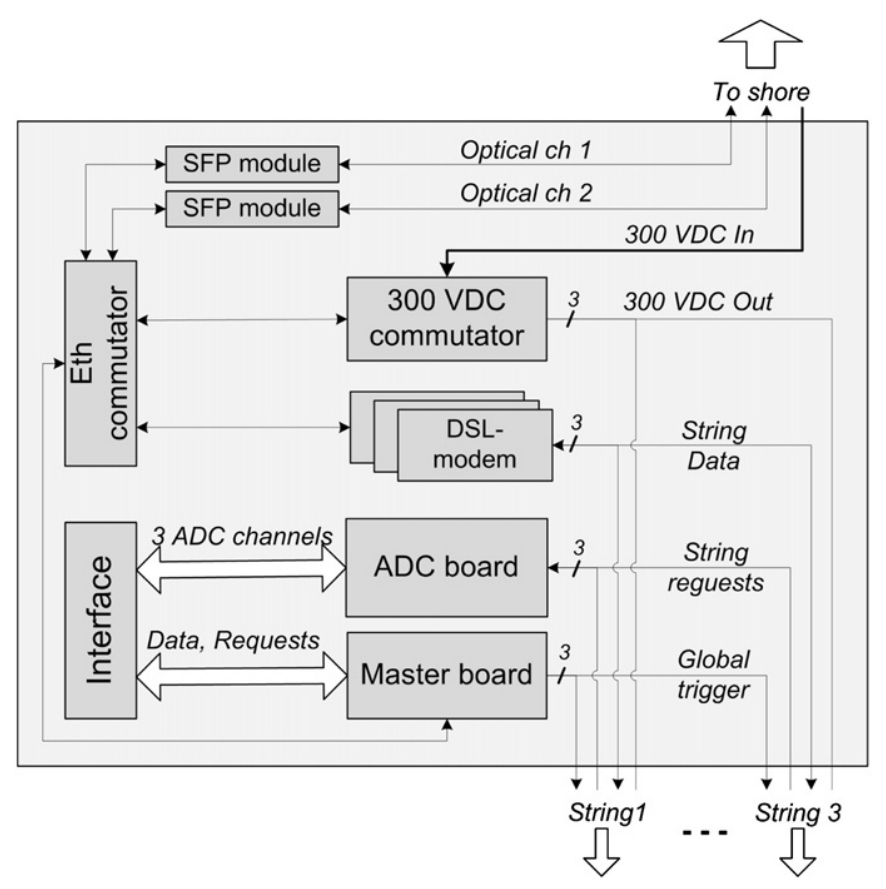

Fig. 13. Block diagram of the cluster DAQ center unit of the 2011 prototype GVD cluster.

Table 1

Average values of reconstructed intensities for five light source output series.

\begin{tabular}{lllrrr}
\hline$I, 10^{12} \gamma /$ pulse & $I_{1}$ & $I_{2}$ & $I_{3}$ & $I_{4}$ & $I_{5}$ \\
\hline Cluster & 64 & 27 & 9.7 & 4.3 & 2.4 \\
NT200 & 63 & 28 & 10.4 & 5.5 & 3.8 \\
\hline
\end{tabular}

The prototype cluster DAQ center is connected to shore by optical Ethernet lines. An electro-optical cable of $6 \mathrm{~km}$ length with three pairs of optical fibers and three copper lines was deployed in 2011.

The prototype cluster is successfully operating now. The cluster DAQ system is being tested with the Baikal underwater calibration laser [12], with LED flashers, and atmospheric muons. Two trigger modes are used for muon detection: four-fold coincidences of optical modules on a string (trigger 4/1, event rate $4 \mathrm{~Hz}$ ), and inter-string two-fold coincidences of two strings (trigger $2 / 2$, event rate $0.5 \mathrm{~Hz}$ ). All tests of the prototype cluster show good performance of the basic cluster elements.

An external calibration laser provides five series of $480 \mathrm{~nm}$ light pulses at five fixed intensity levels ranging from approximately $10^{12}$ to $6 \times 10^{13}$ photons/pulse [12], which corresponds to shower energies from $10 \mathrm{PeV}$ to $600 \mathrm{PeV}$. Distances between the light source and OMs of prototype cluster range from $110 \mathrm{~m}$ up to $180 \mathrm{~m}$ (see Fig. 12). Data simultaneously accumulated by prototype cluster and NT200 were used for the source intensity reconstruction. Results of the reconstruction with the two arrays are presented in Table 1 . Shown are the mean values of reconstructed intensities for five series of the laser.

In Figs. 14 and 15, the distributions of reconstructed intensities obtained from prototype cluster and NT200 data for the first and fourth series are shown. Although the results obtained with two arrays for three highest laser intensities are in a good agreement each other, a relative differences of about $30-50 \%$ were observed for the fourth and fifth laser intensities. This disagreement is caused mainly by a relatively low accuracy of amplitude 


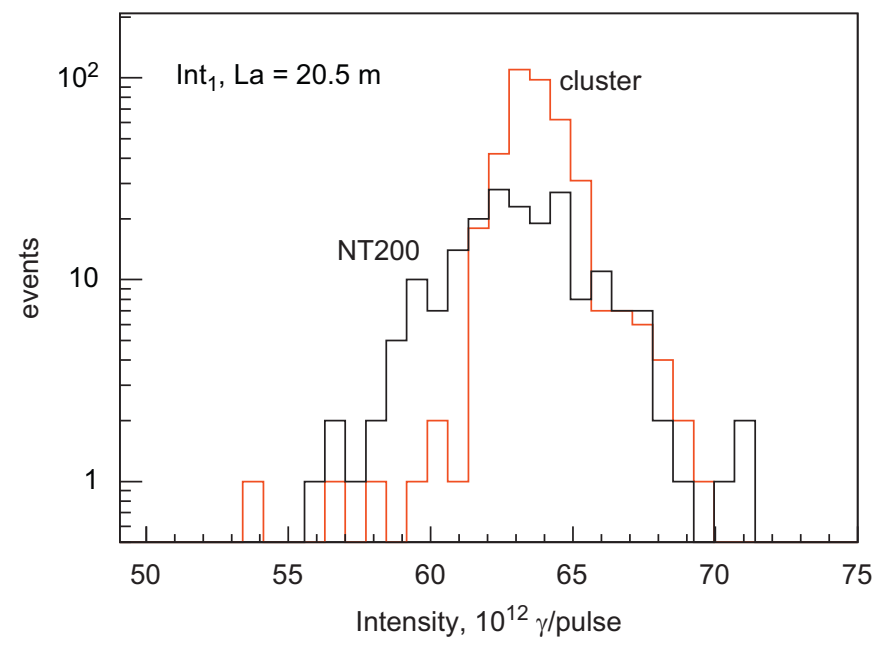

Fig. 14. Reconstructed intensities for the first series.

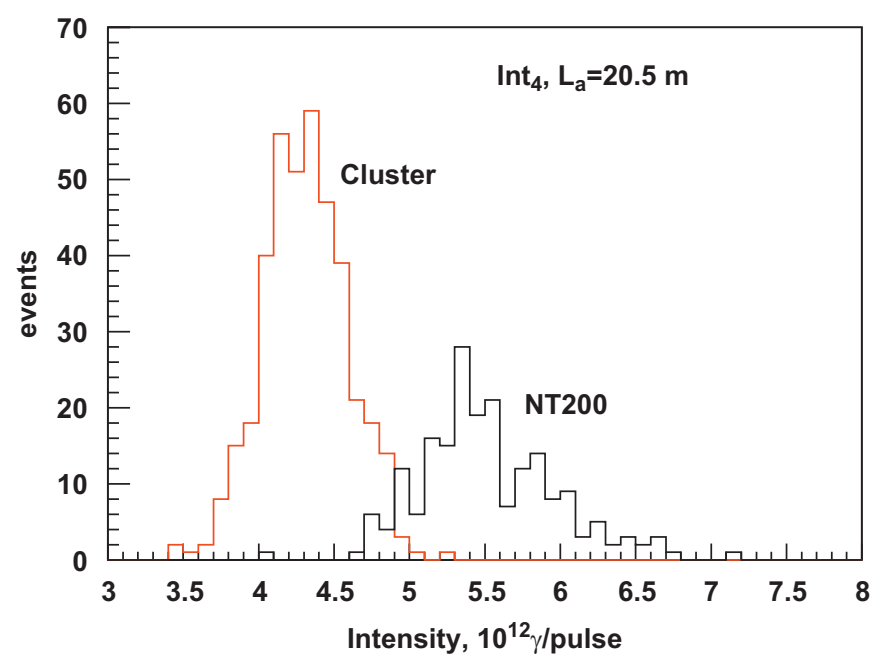

Fig. 15. Reconstructed intensities for the fourth series.

calibration procedure and rather narrow dynamic ranges of optical modules of NT200. Further performance check of prototype cluster measuring systems will be achieved from the ongoing atmospheric muons flux analysis.

\section{Conclusion}

The objective of the Baikal Project is the construction of a kilometer-scale high-energy neutrino observatory: the Gigaton
Volume Detector (GVD) in Lake Baikal. Basic elements of the GVD (new optical modules with PMTs of various types, FADC readout units, underwater communication systems) were investigated and tested in situ with prototype strings in 2008-2010. Measurements with a LED flasher, a calibration laser, and muons allow estimating the time accuracy of the GVD measuring system at the level of about $2 \mathrm{~ns}$. On the basis of the studies of the prototype strings and optical module parameters the optimization of the GVD configuration was performed. The detection area for muons above $3 \mathrm{TeV}$ with arrival direction reconstruction error about of $0.5^{\circ}$ and energy resolution of $\delta \lg E \sim 0.4$ is about $0.1-0.8 \mathrm{~km}^{2}$ (condition 6/3). The effective volume for cascades above $100 \mathrm{TeV}$ with reconstruction error of $5-7^{\circ}$ and energy resolution of about (10-15)\% is $0.1-0.7 \mathrm{~km}^{3}$ (condition 15/3). A technical design report for the GVD Baikal neutrino telescope has been prepared. A prototype cluster which comprises all key elements of the measuring and communication systems of GVD cluster is operating in Lake Baikal since April 2011. In 2012 first full scale GVD string comprising final versions of key elements and electronics will be installed in Lake Baikal.

\section{Acknowledgments}

This work was supported in part by the Russian Ministry of Education and Science (GK 02.740.11.0018, 02.518.11.7158, 16.518.11.7056, 02.740.11.0018, 14.740.11.0890, P133, P691, P878, P1146, P1242, P2504, RNP 2.2.1.1/1483, RNP 2.1.1/1539, RNP 2.2.1.1/5901, REC "BAIKAL"), by the Russian Foundation for Basic Research (grants 11-02-00983, 11-02-10005, 11-02-10017, 11-02-10062).

\section{References}

[1] A.V. Avrorin, et al., Nuclear Instruments and Methods in Physics Research Section A 639 (2011) 30.

[2] A.V. Avrorin, et al., Nuclear Instruments and Methods in Physics Research Section A 626 (2011) 13.

[3] V.M. Aynutdinov, et al., Nuclear Instruments and Methods in Physics Research Section A 588 (2008) 99.

[4] V.M. Aynutdinov, et al., Nuclear Instruments and Methods in Physics Research Section A 567 (2006) 433.

[5] I.A. Belolaptikov, et al., Astroparticle Physics 7 (1997) 263.

[6] V.M. Aynutdinov, et al., Astroparticle Physics 29 (2008) 366.

[7] A.V. Avrorin, et al., Astronomy Letters 35 (2009) 651.

[8] A.V. Avrorin, et al., Astronomy Letters 37 (2011) 692.

[9] R. Bagduev, et al., Nuclear Instruments and Methods in Physics Research Section A 420 (1999) 138.

[10] A.V. Avrorin, et al., Instruments and Experimental Techniques 54 (2011) 649.

[11] V.M. Aynutdinov, et al., Astroparticle Physics 25 (2006) 140.

[12] V.M. Aynutdinov, et al., Physics of Atomic Nuclei 69 (2006) 1914. 